Supplement of E\&G Quaternary Sci. J., 69, 165-186, 2020

https://doi.org/10.5194/egqsj-69-165-2020-supplement

(c) Author(s) 2020. This work is distributed under

the Creative Commons Attribution 4.0 License.

(c) (1)

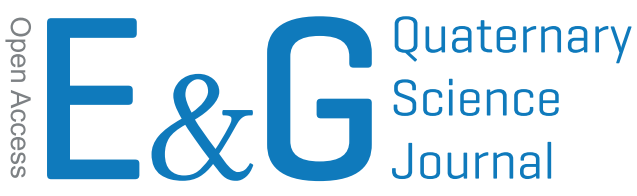

Supplement of

\title{
Reconstruction of palaeoenvironmental variability based on an inter-comparison of four lacustrine archives on the Peloponnese (Greece) for the last 5000 years
}

Joana Seguin et al.

Correspondence to: Joana Seguin (jseguin@ecology.uni-kiel.de)

The copyright of individual parts of the supplement might differ from the CC BY 4.0 License. 


\section{Supplementary Online Material}

Figure S1: Updated Bayesian age-depth model for Asea. The model was constructed using the $\mathrm{R}$ package rbacon (Blaauw and Christen, 2011). The blue tie bars indicate the ${ }^{14} \mathrm{C}$ age distributions. Outliers are plotted in red and excluded from modelling. The greyscale of the line graph reflects the likelihood; the darker the more likely the model passes through that age. The red dotted line follows the mean ages. For the initial age-depth-model please refer to Unkel et al. (2014).

\section{Figure S2: Comparison of uncertainty ranges of age-depth-models for all four sites.}

Figure S3: Comparison of $\log (\mathrm{Ca} / \mathrm{Ti})$ (left y-axes) and PC1 (right $\mathrm{y}$-axes) proxies for all study sites. (a) Stymphalia, (b) Kaisari, (c) Pheneos, (d) Asea. For orientation, boxes with cultural periods (Table S6) are plotted on top. For the interpretation, the reader is referred to the text.

Table S1: Sedimentary units of core PHE1. The soil type classification follows the German ad-hoc Arbeitsgruppe Boden (2005); (UB = upper boundary, G = gradational).

Table S2: Sedimentary units of core KES2. The soil type classification follows the German ad-hoc Arbeitsgruppe Boden (2005); (UB = upper boundary, G = gradational).

Table S3: Cultural chronology of southern Greece (slightly modified after Weiberg et al., 2016; Bintliff, 2012; Manning, 2010). 


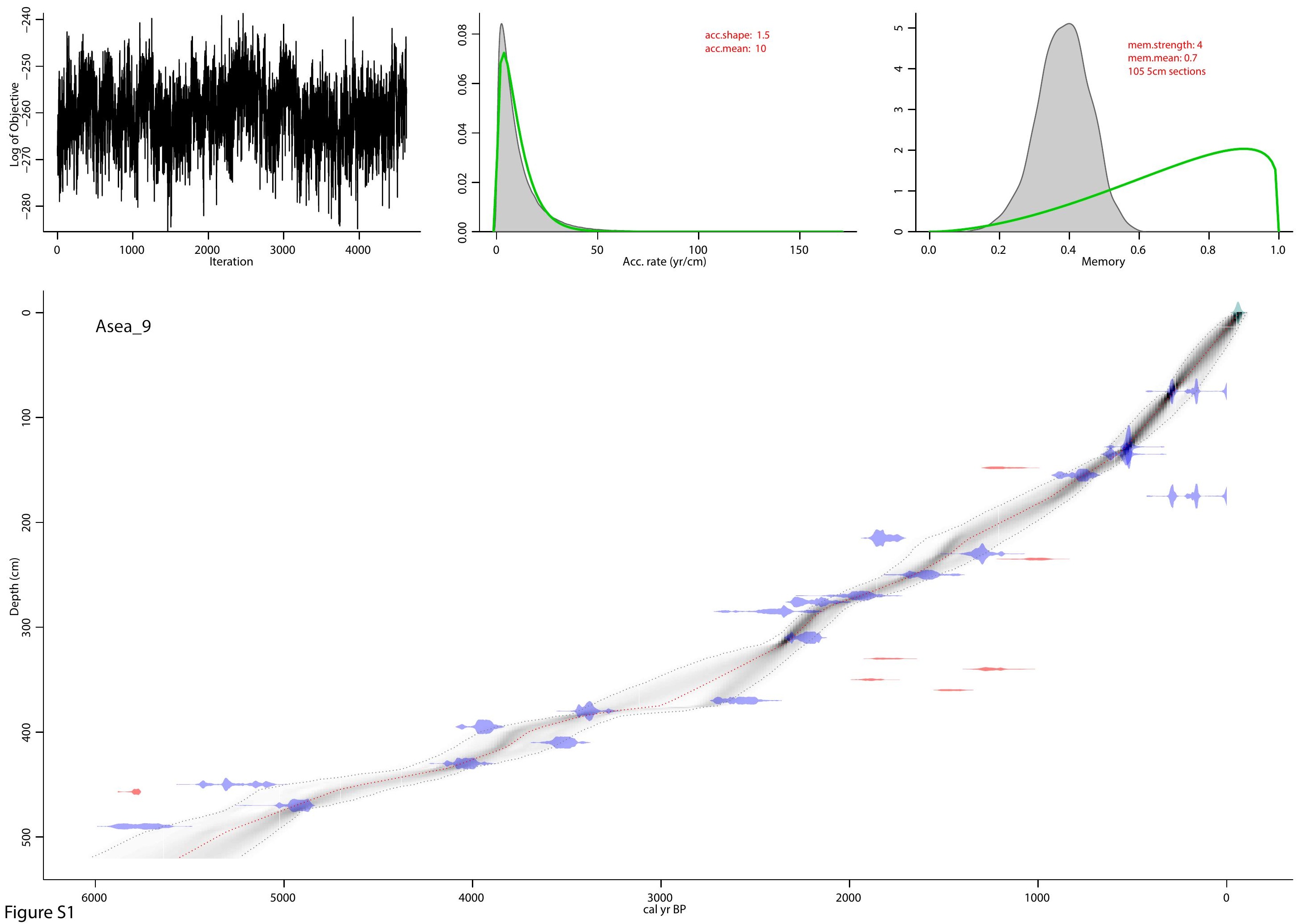


(a) STY1

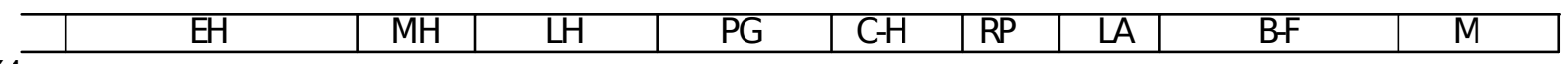

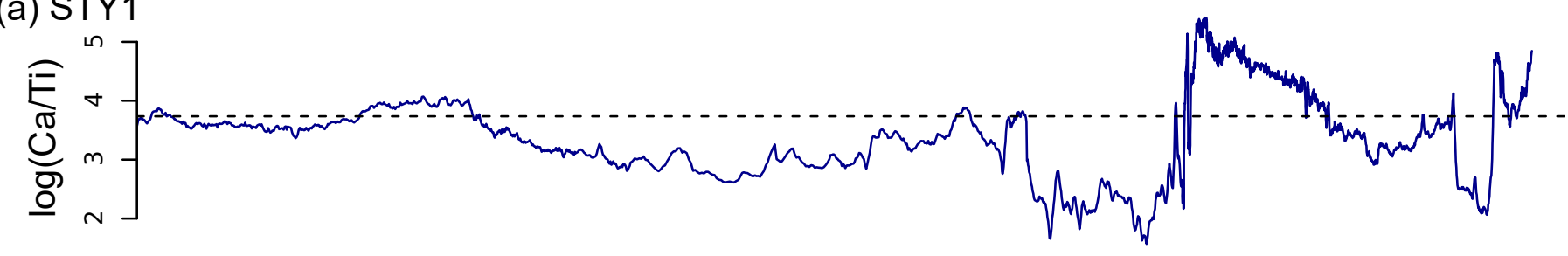

(b) KES2

2

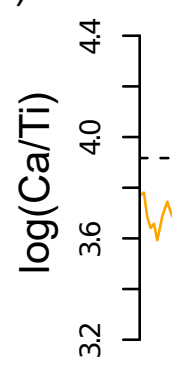

(c) PHE1

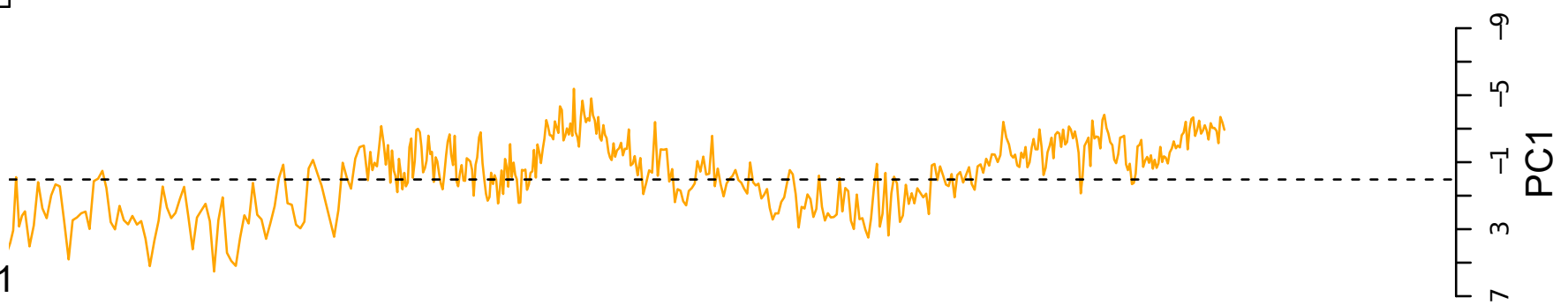

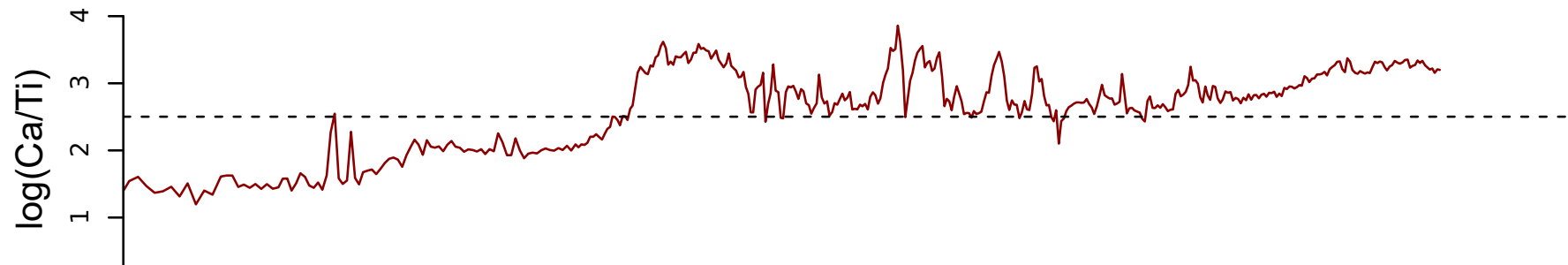

(d) Asea $\mathrm{NhmWMr}$

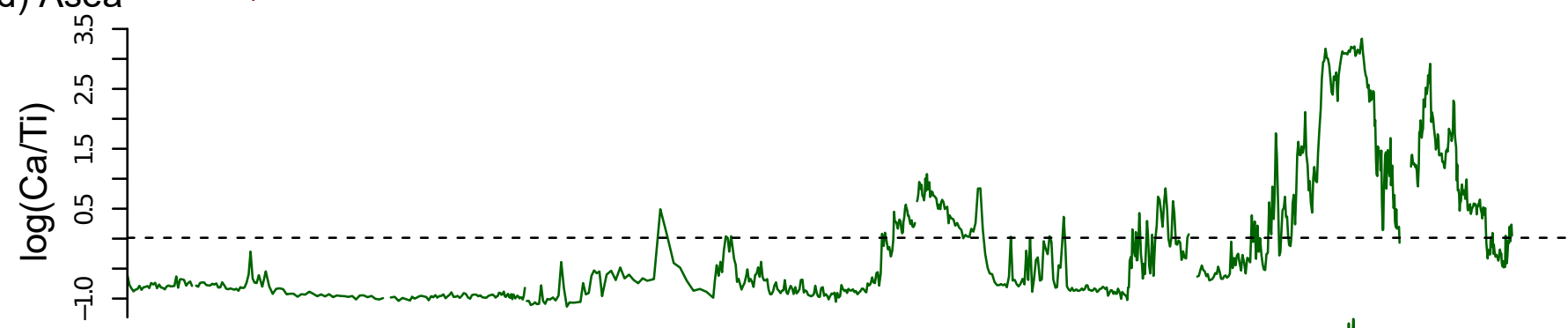

$$
\text { 2000 }
$$

Figure S3

\begin{tabular}{llllll}
\hline 5000 & 4000 & 3000 & 2000 & 1000 & 0
\end{tabular}


Table S1: Sedimentary units of core PHE1. The soil type classification follows the German ad-hoc Arbeitsgemeinschaft Boden (2005).

(UB = upper boundary, $\mathrm{G}=$ gradational)

\begin{tabular}{|c|c|c|c|c|c|}
\hline $\begin{array}{l}\text { Core } \\
\text { Depth } \\
{[\mathrm{cm}]}\end{array}$ & $\begin{array}{c}\text { Sediment } \\
\text { Unit }\end{array}$ & $\begin{array}{c}\text { Unit } \\
\text { Thickness } \\
\text { [cm] }\end{array}$ & $\begin{array}{l}\text { Munsell } \\
\text { Color }\end{array}$ & Soil Type & Description \\
\hline $0-46$ & 9 & 46 & $\begin{array}{l}10 \mathrm{YR} 5 / 3 \\
\text { brown }\end{array}$ & Tu4 & $\begin{array}{l}\text { homogeneous brown, hardly mottled, well } \\
\text { mixed, very compact, plough horizon } \\
\rightarrow \text { excluded from analysis }\end{array}$ \\
\hline $46-83$ & 8 & 37 & $\begin{array}{l}10 Y R 4 / 4 \\
\text { brown } \\
\text { (slight } \\
\text { greyish } \\
\text { touch) }\end{array}$ & Ut3 & $\begin{array}{l}\text { similar to unit } 7 \text { without red colour, few shell } \\
\text { fragments, fewer blackish mottles and more } \\
\text { homogeneous than unit } 7 \text {, blackish clasts at } \\
83-73 \mathrm{~cm},(\mathrm{UB}=\mathrm{G})\end{array}$ \\
\hline $83-156$ & 7 & 73 & $\begin{array}{l}\text { 10YR 4/3 } \\
\text { brown }\end{array}$ & Ut4 & $\begin{array}{l}\text { slightly brighter than unit } 6 \text { but with reddish } \\
\text { patches (decreasing towards top), slightly } \\
\text { more blackish/charcoal flitter, few shell } \\
\text { fragments (gastropods), absence of clasts - } \\
\text { only fine matrix, (UB = very G) }\end{array}$ \\
\hline $156-192$ & 6 & 36 & $\begin{array}{l}\text { 10YR 4/3 } \\
\text { brown }\end{array}$ & Ut4 & $\begin{array}{l}\text { yellow-grey-mottled, fine charcoal particles } \\
\text { intensely visible up to } 180 \mathrm{~cm} \text {, absence of } \\
\text { clasts - only fine matrix, very few shell } \\
\text { fragments, }(\mathrm{UB}=\mathrm{G})\end{array}$ \\
\hline $192-235$ & 5 & 43 & $\begin{array}{l}10 \text { YR } 3 / 2 \\
\text { very dark } \\
\text { greyish } \\
\text { brown }\end{array}$ & Ut3 & $\begin{array}{l}\text { sub-angular clasts of up to } 1 \mathrm{~cm} \text { diameter } \\
\text { decreasing towards top, sediment getting } \\
\text { brighter, continuous charcoal particles, very } \\
\text { tiny shell fragments, }(\mathrm{UB}=\mathrm{G})\end{array}$ \\
\hline $235-300$ & 4 & 65 & $\begin{array}{l}10 \mathrm{YR} 4 / 3 \\
\text { dark } \\
\text { yellowish } \\
\text { brown }\end{array}$ & Ut4 & $\begin{array}{l}\text { fewer clasts but darker colour and larger than } \\
\text { in unit } 2+3 \text {, all clasts are subangular, colour is } \\
\text { similar to unit } 3 \text { with lots of orange mottles } \\
\text { and gets slightly darker towards top, } \\
\text { continuous charcoal particles/slightly fewer } \\
\text { than in unit } 3 \text {, some very tiny shell fragments, } \\
\text { (UB = very G) }\end{array}$ \\
\hline $300-329$ & 3 & 29 & $\begin{array}{l}10 \mathrm{YR} 4 / 3 \\
\text { or } 4 / 4 \text { dark } \\
\text { yellowish } \\
\text { brown }\end{array}$ & Ut4 & $\begin{array}{l}\text { more abundant small, in situ formed } \\
\text { carbonate nodules }<55 \mathrm{~mm} \text { than in unit } 2 \text {, } \\
\text { clay matrix but less clayey than in unit } 2 \text { - } \\
\text { dull surface (similar to unit } 2 \text { but brighter } \\
\text { colour), water holes at } 3.28 \text { and } 3.23 \mathrm{~m} \text {, } \\
\text { orange patches e.g. } 3.46 \mathrm{~m} \text {, darker in central } \\
\text { part of the unit, }(\mathrm{UB}=\mathrm{G} \text { ) }\end{array}$ \\
\hline $329-360$ & 2 & 31 & $\begin{array}{l}10 \text { YR 3/1 } \\
\text { very dark } \\
\text { grey }\end{array}$ & Ut4 & $\begin{array}{l}\text { clayey matrix, darkest unit, occasional orange } \\
\text { spots (oxidation), occasional gravel clasts } \\
\text { with max. } 5 \mathrm{~mm} \text { diameter, } 346-360 \mathrm{~cm} \text { : } \\
\text { minor charcoal particles, }(\mathrm{UB}=\mathrm{G})\end{array}$ \\
\hline $360-390$ & 1 & 30 & $\begin{array}{l}\text { 10YR 4/4 } \\
\text { dark } \\
\text { yellowish } \\
\text { brown }\end{array}$ & Ut3 & $\begin{array}{l}\text { mix of grey-brown-yellow and white-orange- } \\
\text { oxidized mottles, brightest unit, fine matrix } \\
\text { with clasts and carbonate nodules of up to } \\
1 \mathrm{~cm} \text { diameter, clasts are subangular and } \\
\text { unsorted, gravel content decreases upwards, } \\
\text { increase in organic content (= black colour } \\
\text { intensifies, charcoal flitter visible), fining } \\
\text { upwards, (UB = G) }\end{array}$ \\
\hline
\end{tabular}


Table S2: Sedimentary units of core KES2. The soil type classification follows the German ad-hoc Arbeitsgemeinschaft Boden (2005).

(UB = upper boundary, $\mathrm{G}=$ gradational)

\begin{tabular}{|c|c|c|c|c|c|}
\hline $\begin{array}{l}\text { Core } \\
\text { Depth } \\
{[\mathrm{cm}]}\end{array}$ & $\begin{array}{l}\text { Sediment } \\
\text { Unit }\end{array}$ & $\begin{array}{l}\text { Unit } \\
\text { Thickness } \\
\text { [cm] }\end{array}$ & $\begin{array}{l}\text { Munsell } \\
\text { Color }\end{array}$ & Soil Type & Description \\
\hline $0-50$ & 7 & 50 & $\begin{array}{l}10 Y R \text { R/4 } \\
\text { dark } \\
\text { yellowish } \\
\text { brown }\end{array}$ & Ut4 & $\begin{array}{l}\text { plough horizon, plant remains and roots in } \\
\text { upper } 15 \mathrm{~cm} \text { visible, core segment strongly } \\
\text { damaged - difficult to delimit boundaries } \\
\rightarrow \text { excluded from analysis }\end{array}$ \\
\hline $50-125$ & 6 & 75 & $\begin{array}{l}\text { 10YR 5/4 } \\
\text { yellowish } \\
\text { brown }\end{array}$ & Ut4 & $\begin{array}{l}\text { similar to unit } 5 \text { but black speckles become } \\
\text { fewer, gets brighter towards top, less mottled, } \\
\text { silt content increasing upwards, patches of } \\
\text { red soil and brick/loam? (e.g. } 1.10 \mathrm{~m} ; 1.00 \mathrm{~m} \text {; } \\
0.87 \mathrm{~m}) \text {, unit becomes brighter towards top, } \\
(\mathrm{UB}=\mathrm{G})\end{array}$ \\
\hline $125-165$ & 5 & 40 & $\begin{array}{l}2.5 \mathrm{Y} 4 / 3 \\
\text { olive } \\
\text { brown }\end{array}$ & Ut3 & $\begin{array}{l}\text { darker olive-grey colour, black speckles, } \\
\text { higher organic content, strongly mottled, } \\
(\mathrm{UB}=\mathrm{G})\end{array}$ \\
\hline $165-205$ & 4 & 40 & $\begin{array}{l}2.5 \mathrm{Y} 6 / 4 \\
\text { light } \\
\text { yellowish } \\
\text { brown }\end{array}$ & Ut4 & $\begin{array}{l}\text { brightest unit of the core, yellowish-beige } \\
\text { colour, especially visible in } 2 \mathrm{c} \text { while } 2 \mathrm{~b} \\
\text { resembles more unit } 3 \text {, unit has a very } \\
\text { different morphostructure: matt, airy, very } \\
\text { high carbonate content, (UB=seepage water } \\
\text { shift?) }\end{array}$ \\
\hline $205-240$ & 3 & 35 & $\begin{array}{l}10 Y R 5 / 3 \\
\text { brown }\end{array}$ & Ut4 & $\begin{array}{l}\text { similar to unit } 2 \text { but less reddish instead more } \\
\text { greyish colour, high carbonate content, few } \\
\text { shell fragments }(\mathrm{UB}=\mathrm{G})\end{array}$ \\
\hline $240-310$ & 2 & 70 & $\begin{array}{l}7.5 \mathrm{YR} 4 / 4 \\
\text { brown }\end{array}$ & Ut4 & $\begin{array}{l}\text { reddish colour dominates, contains most } \\
\text { black speckles in whole core sequence, very } \\
\text { strongly mottled, very clayey adhesive but } \\
\text { matt surface, no shell fragments visible, } \\
(\mathrm{UB}=\mathrm{G})\end{array}$ \\
\hline $310-350$ & 1 & 40 & $\begin{array}{l}2.5 \mathrm{Y} 5 / 3 \\
\text { light olive } \\
\text { brown }\end{array}$ & Ut3 & $\begin{array}{l}\text { matrix beige-yellowish-greenish-gley } \\
\text { shimmer with black and yellow-orange } \\
\text { speckles, some shell fragments throughout the } \\
\text { unit, black spots look finely dotted, }(\mathrm{UB}=\mathrm{G})\end{array}$ \\
\hline
\end{tabular}


Table S3. Cultural chronology of southern Greece (slightly modified after Weiberg et al., 2016; Bintliff, 2012; Manning, 2010).

\begin{tabular}{|c|c|c|c|c|}
\hline Time (BP) & Time (BC/AD) & Periods & Event & Abbreviation \\
\hline $490--60$ & AD $1460-2010$ & $\begin{array}{l}\text { Ottoman Empire to } \\
\text { Modern Period }\end{array}$ & $\begin{array}{l}\text { Start: Ottoman conquest } \\
\text { of the Peloponnese }\end{array}$ & M \\
\hline $1309-490$ & AD $641-1460$ & $\begin{array}{l}\text { Medieval: } \\
\text { Early Byzantine to } \\
\text { Byzantine-Frankish }\end{array}$ & $\begin{array}{l}\text { Start: Death of Emperor } \\
\text { Heraclius and the collapse } \\
\text { of the Late Roman } \\
\text { political order. }\end{array}$ & $\mathrm{B}-\mathrm{F}$ \\
\hline $1650-1309$ & AD $300-641$ & $\begin{array}{l}\text { Late Antiquity/ } \\
\text { Late Roman }\end{array}$ & $\begin{array}{l}\text { Start: Founding of the city } \\
\text { of Constantinople and the } \\
\text { parting of ways between } \\
\text { the Western and Eastern } \\
\text { parts of the Roman } \\
\text { Empire. }\end{array}$ & LA \\
\hline $1981-1650$ & $\begin{array}{l}31 \mathrm{BC}-\mathrm{AD} \\
300\end{array}$ & Roman & $\begin{array}{l}\text { Start: Destruction of } \\
\text { Corinth and end of } \\
\text { Achaian war. }\end{array}$ & $\mathrm{RP}$ \\
\hline $2429-1981$ & $479-31 \mathrm{BC}$ & $\begin{array}{l}\text { Classical, } \\
\text { Hellenistic }\end{array}$ & $\begin{array}{l}\text { Start: Greek victory over } \\
\text { the Persians in the battle } \\
\text { of Plataea; Persian } \\
\text { invasion of Greece } \\
\text { repelled. }\end{array}$ & $\mathrm{C}-\mathrm{H}$ \\
\hline $3025-2429$ & $1075-479 \mathrm{BC}$ & $\begin{array}{l}\text { Proto-Geometric, } \\
\text { Early Iron Age, } \\
\text { Archaic }\end{array}$ & $\begin{array}{l}\text { Start: End of Mycenaean } \\
\text { culture }\end{array}$ & PG \\
\hline $3650-3025$ & $1700-1075 \mathrm{BC}$ & $\begin{array}{l}\text { Late } \\
\text { Helladic/Mycenean }\end{array}$ & $\begin{array}{l}\text { Periodization modelled on } \\
\text { Minoan Crete, which in } \\
\text { turn was modelled on the } \\
\text { Old, } \\
\text { Middle and New } \\
\text { Kingdom of ancient } \\
\text { Egypt }\end{array}$ & LH \\
\hline $4050-3650$ & $2100-1700 \mathrm{BC}$ & Middle Helladic & & $\mathrm{MH}$ \\
\hline $5050-4050$ & $3100-2100 \mathrm{BC}$ & Early Helladic & $\begin{array}{l}\text { Beginning of the Bronze } \\
\text { Age }\end{array}$ & $\mathrm{EH}$ \\
\hline $6450-5050$ & $4500-3100 \mathrm{BC}$ & Final Neolithic & $\begin{array}{l}\text { Start: Introduction of a } \\
\text { farming economy in } \\
\text { Greece }\end{array}$ & FN \\
\hline $7400-6450$ & $5450-4500 \mathrm{BC}$ & Late Neolithic & & $\mathrm{LN}$ \\
\hline $7900-7400$ & $5950-5450 \mathrm{BC}$ & Middle Neolithic & & $\mathrm{MN}$ \\
\hline $8750-7900$ & $6800-5950 \mathrm{BC}$ & $\begin{array}{l}\text { Initial and Early } \\
\text { Neolithic }\end{array}$ & & EN \\
\hline
\end{tabular}

